\title{
Bound states can stabilize electroweak strings
}

\author{
Tanmay Vachaspati \\ Tufts Institute of Cosmology, Department of Physics and Astronomy, Tufts University, Medford, MA 02155, USA
}

and

Richard Watkins

Physics Department, University of Michigan, Ann Arbor, MI 48109, USA

Received 8 June 1993; revised manuscript received 30 August 1993

Editor: H. Georgi

\begin{abstract}
We show that the stability of the electroweak $Z$-string is greatly improved by the presence of bound states of a complex scalar field. This stabilization mechanism could work for other embedded defects and also for unstable solutions such as the sphaleron.
\end{abstract}

It is now known that vortex solutions [1] may be embedded in almost any field theoretic model that exhibits spontaneous symmetry breaking [2]. In particular, two distinct vortex solutions are known to be embedded in the standard electroweak model [2-5]. These are called the $W$-string and the $Z$-string in the literature. The $W$-string is conjectured to be unstable for all values of the parameters while the $Z$-string has been shown to be stable only for $\sin ^{2} \theta_{W} \approx 1$. The $Z$-string in the standard electroweak model with $\sin ^{2} \theta_{W}=0.23$ is unstable [6,7] and remains so even at high temperatures such as would be present in the early universe [8].

If the string solutions are indeed unstable under all circumstances, their relevance to physical processes would probably be negligible. However, in this letter we shall show that the strings can be stabilized by the presence of other scalar (and perhaps fermionic) fields in the theory. The idea behind this result is quite simple to understand, especially if one is aware of the reason that permits the existence of non-topological solitons [9]. Suppose that we have a theory in which the Higgs mechanism is responsible for generating the mass of a certain scalar. Then, after the symmetry breaking, the Higgs field gives the scalar a mass but the back-reaction of the scalar field on the Higgs field is to try and prevent the Higgs field from acquiring its vacuum-expectation-value (VEV). In other words, the scalar would rather live in a region where the Higgs field vanishes since the mass of the scalar field is zero wherever the Higgs field is zero. But the center of the string is precisely a region where the Higgs field vanishes. Therefore the scalar likes to accumulate on the string and tends to maintain the string configuration with its region of vanishing Higgs field - that is, the scalar adds to the stability of the configuration. Yet another way of stating this idea is that the string is a "bag" in which the scalar prefers to sit and, hence, hold together.

In what follows, we shall only consider the case of a scalar field interacting with the electroweak $Z$ string. To start with, we shall describe the effect of scalar bound states on semilocal strings $[10]$ where it is fairly clear that the stability improves due to the bound state. This in itself shows that the electroweak $Z$-string will become more stable when it has scalar bound states since, after all, the $Z$-string is nothing but the semilocal string when $\sin ^{2} \theta_{W}=1$. However, we go further and explicitly examine the case of the $Z$-string with a scalar bound state. Our results suggest that it may be possible to get stable $Z$-strings even when $\sin ^{2} \theta_{W}=0.23$. 
This does not immediately imply that stable $Z$ strings occur in the standard electroweak model since there is no extra scalar field in this model. However, the standard model does contain leptons and quarks which will also have bound states on the string. We expect the arguments of the previous paragraphs to apply in this case too since, once again, it is favorable for the fermion to sit in the string "bag" and to prevent the bag from decaying. We note, however, that fermions can be expected to be somewhat less efficient at stabilizing strings due to the exclusion principle. Whether the lepton and quark bound states are sufficient to stabilize the $Z$-string is another story that needs detailed investigation. We hope to undertake this task in the near future.

The Lagrangian that yields semilocal string solutions with an additional complex scalar field is

$$
\begin{aligned}
L_{\mathrm{sl}} & =\left(\mathrm{D}^{\mu} \phi\right)^{\dagger}\left(\mathrm{D}_{\mu} \phi\right)+\left(\partial^{\mu} \chi\right)^{*}\left(\partial_{\mu} \chi\right)-\frac{1}{4} F_{\mu \nu}^{Z} F_{Z}^{\mu \nu} \\
& -V(\phi, \chi),
\end{aligned}
$$

where,

$$
\begin{aligned}
& V(\phi, \chi)=\lambda_{1}\left(\phi^{\dagger} \phi-\frac{1}{2} \eta^{2}\right)^{2}+\lambda_{2}|\chi|^{4} \\
& \quad+2 \lambda_{3}\left(\phi^{\dagger} \phi \pm m^{2}\right) \chi^{*} \chi .
\end{aligned}
$$

The field $\phi$ is a global SU (2) doublet carrying a gauged $\mathrm{U}(1)$ charge, while $\chi$ is a single complex field. The covariant derivative is defined by

$\mathrm{D}_{\mu}=\partial_{\mu}+\frac{1}{2} \mathrm{i} \alpha Z_{\mu}$.

There are two approaches to finding solutions that describe a string with a non-trivial $\chi$ configuration. The first is that, for the negative sign in (2) and for some values of the parameters, the string configuration together with $\chi=0$ is unstable, and the stable ground state solution is one that has a non-trivial $\chi$ condensate on the string [11]. It may be speculated that the presence of a condensate ${ }^{\# 1}$ might improve the stability of the string. Indeed we have checked that there is an improvement in string stability due to a condensate but the improvement is only marginal and

\#1 Note the distinction between "condensate" and "bound state". A condensate is the ground state configuration of $\chi$ in the background of the string while a bound state requires the presence of $\chi$ particles. is certainly not enough to stabilize the string when $\sin ^{2} \theta_{W}=0.23$. The second approach is to consider the string in the presence of $\chi$ particles - that is, the string with $\chi$ bound states. The $\chi$ particles carry a conserved $U(1)$ global charge which is derived from the conserved current

$j^{\mu}=\frac{1}{2} \mathrm{i}\left(\chi^{*} \partial^{\mu} \chi-\chi \partial^{\mu} \chi^{*}\right)$.

Hence, we consider a string in the presence of a definite amount of global $U(1)$ charge. This, together with cylindrical symmetry, leads to the following ansatz for $\chi$ :

$\chi=\mathrm{e}^{\mathrm{i} \omega t} \psi(r)$,

where $r$ is the cylindrical radial coordinate. The charge per unit length along the $z$-direction in this configuration is

$q=2 \pi \omega \int \mathrm{d} r r \psi^{2}$

We will look at solutions of the equations of motion following from (1) that consist of a semilocal string and a fixed amount of $U(1)$ charge. In accordance with the usual ansatz for the semilocal string [10], we take

$\phi=\left(\begin{array}{c}0 \\ f(r) \mathrm{e}^{\mathrm{i} \theta}\end{array}\right), \quad Z_{\mu}=-\frac{v(r)}{r} \hat{\boldsymbol{e}}_{\theta}$.

It is now convenient to rescale the fields and coordinates to make them dimensionless:

$P=\frac{\sqrt{2}}{\eta} f, \quad V=\frac{\alpha}{2} v, \quad w=\frac{4 \sqrt{\lambda_{3}}}{\alpha \eta} \psi$,

$R=\frac{\alpha \eta}{2 \sqrt{2}} r$.

Then the equations of motion are

$P^{\prime \prime}+\frac{P^{\prime}}{R}-(1-V)^{2} \frac{P}{R^{2}}+\beta\left(1-P^{2}\right) P-w^{2} P=0$,

$V^{\prime \prime}-\frac{V^{\prime}}{R}+2(1-V) P^{2}=0$,

$w^{\prime \prime}+\frac{w^{\prime}}{R}-\lambda w^{3}-\gamma\left(P^{2}-\delta^{2}\right) w=0$, 
where primes denote derivatives with respect to $R$. The parameters entering these equations are defined by

$$
\begin{aligned}
& \beta=\frac{8 \lambda_{1}}{\alpha^{2}}=\frac{m_{H}^{2}}{m_{Z}^{2}}, \quad \lambda=\frac{\lambda_{2}}{\lambda_{3}}, \quad \gamma=\frac{8 \lambda_{3}}{\alpha^{2}}=\frac{m_{\chi}^{2}}{m_{Z}^{2}}, \\
& \delta^{2}=\frac{2}{\eta^{2}}\left(m^{2}+\frac{\omega^{2}}{2 \lambda_{3}}\right) .
\end{aligned}
$$

Here $m_{H}, m_{Z}$ and $m_{\chi}$ denote the masses of the $\phi, Z$ and $\chi$ particles respectively. In addition to eqs. (9), (10) and (11), we also have the constraint that the rescaled (dimensionless) charge is some fixed nonzero constant. Therefore,

$\bar{q} \equiv \frac{\omega}{\eta \sqrt{\lambda_{3}}} \int \mathrm{d} R R[w(R)]^{2}=$ constant.

The boundary conditions on $P, V$ and $w$ are $P(0)=$ $0, P(\infty)=1, V(0)=0, V(\infty)=1, w^{\prime}(0)=0$ and $w(\infty)=0$.

So far we have been looking at the unperturbed string plus bound state solution. Now we turn to the stability analysis.

The stability analysis of the semilocal string [12] can be reduced to an analysis of the perturbation in the upper component of $\phi$ alone. Even in the presence of a bound state, this remains true since it is the upper component of $\phi$ which provides a channel for the string to unwind on the vacuum manifold. If the upper component of $\phi$ was forced to remain zero, the semilocal string would be identical to the NielsenOlesen string which we know to be topologically stable even in the presence of other fields. Hence, it is sufficient to examine perturbations in $\phi_{1}$ - the rescaled upper component of $\phi$. Furthermore, it is sufficient to consider $\phi_{1}$ to be real and a function of the radial coordinate alone $[13,6]$.

The energy variation due to the perturbation $\phi_{1}$ is

$$
\delta E=\eta^{2} \pi \int \mathrm{d} R R\left[{\phi_{1}^{\prime 2}}^{2}+M^{2}(R) \phi_{1}^{2}\right],
$$

where

$$
M^{2}(R)=\frac{V^{2}}{R^{2}}+\beta\left(P^{2}-1\right)+w^{2} .
$$

It is immediately obvious that the presence of a bound state improves the stability of the semilocal string since the contribution to $M^{2}$ coming from $w$ is always positive. In the absence of a bound state, we know that the semilocal string is stable only for $[13,14]$ $0 \leqslant \beta \leqslant 1$. Hence, a bound state on the semilocal string will stabilize the string for values of $\beta$ larger than 1. A quantitative statement about the stability of the "bound semilocal string", however, requires a numerical analysis since the bound state will also backreact on the unperturbed string configuration. Here, since we are primarily interested in the electroweak string, we simply remark that our numerical analysis confirms that the semilocal string can be stabilized for $\beta>1$ if we include a suitable bound state on the string.

The electroweak string will have the same field configuration as the semilocal string, with all the gauge fields except the $Z$ set to zero. To analyze its stability, we use the results of ref. [6], where it is shown that the stability issue reduces to asking if there are any negative eigenvalues $(\Omega)$ to the Schrödinger equation:

$-\frac{1}{R} \frac{\mathrm{d}}{\mathrm{d} R}\left(\frac{R}{Q} \frac{\mathrm{d} \zeta}{\mathrm{d} R}\right)+U(R) \zeta=\Omega \zeta$,

with

$$
\begin{aligned}
U(R) & =\frac{1}{Q} \frac{P^{\prime 2}}{P^{2}}+\frac{2 S}{R^{2} P^{2}}+\frac{1}{R} \frac{\mathrm{d}}{\mathrm{d} R}\left(\frac{R}{Q} \frac{P^{\prime}}{P}\right), \\
Q & =\left(1-2 \cos ^{2} \theta_{W} V\right)^{2}+2 \cos ^{2} \theta_{W} R^{2} P^{2}, \\
S & =\frac{P^{2}}{2}-\cos ^{2} \theta_{W} \frac{V^{\prime 2}}{Q} \\
& +\frac{R}{2} \frac{\mathrm{d}}{\mathrm{d} R}\left(\frac{V^{\prime}}{R} \frac{1-2 \cos ^{2} \theta_{W} V}{Q}\right) .
\end{aligned}
$$

The boundary conditions on $\zeta$ are: $\zeta(0)=1, \zeta^{\prime}(0)=$ 0 and $\zeta(\infty)=0$.

We have first found the unperturbed configuration using (9), (10) and (11) subject to the constraint (13) by using numerical relaxation techniques. Then we have solved (16) by a numerical shooting method which allows us to check if $\Omega$ is positive or negative. In all our numerical work we have taken $\beta=0.40$ $\left(m_{H}=58 \mathrm{GeV}\right)$ - this satisfies the experimental constraint $\beta>0.38$ obtained by LEP. In fig. 1 we show the minimum value of $\sin ^{2} \theta_{W}$ that is required for a string with a certain amount of charge to be stable in the case when $\lambda=0, \gamma=1$ and $m=0,2$. The plot is not very sensitive to the value of $\gamma$ and the value of 


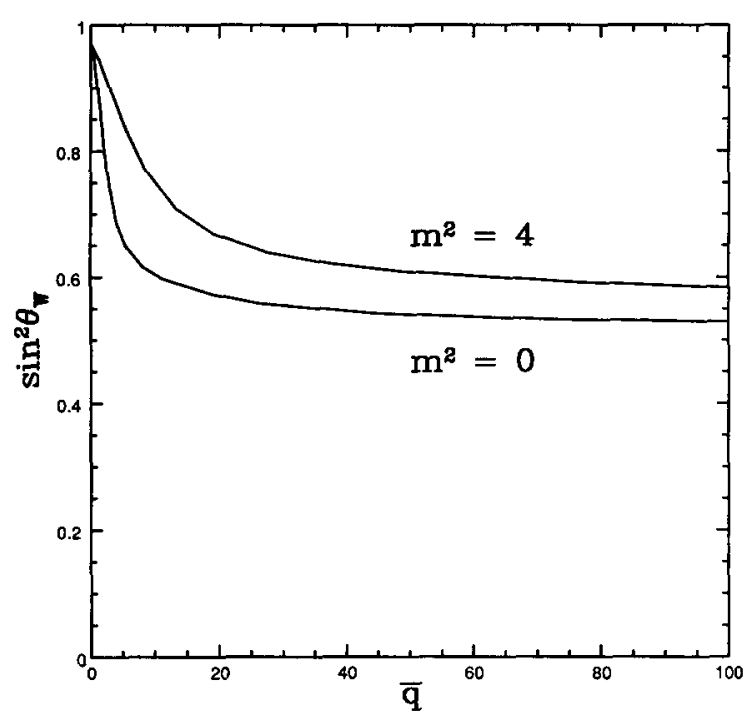

Fig. 1. The value of $\sin ^{2} \theta_{W}$ above which strings with a certain amount of charge $\bar{q}$ are stable plotted versus $\bar{q}$ when $\beta=0.4, \lambda=0, \gamma=1$ and $m=0$ and 2 .

$\sin ^{2} \theta_{W}$ for large $\bar{q}$ depends only weakly on $m$. This plot shows that the stability of the electroweak $Z$-string greatly improves in the presence of bound states and stability at lower values of $\sin ^{2} \theta_{W}$ may be achieved by putting enough charge on the string. However, our numerical analysis was not able to find a stable solution for the physical case $\sin ^{2} \theta_{W}=0.23$. We do not know if a more extensive exploration of parameter space or the addition of more charge would result in a stable solution in this case also or if there is some hard lower bound on $\sin ^{2} \theta_{W}$ which cannot be crossed.

The reader may wonder if the introduction of the field $\chi$ could have introduced some new instability in the configuration. As was shown in ref. [15] in the case of cylindrical non-topological solitons, there is a possible instability in the distribution of charge along the string - the linear distribution of scalar charge may be unstable to clumping up in spherical regions. It is easy to see how this instability can arise. Consider the energy per unit length of a $z$-independent string configuration as a function of its charge per unit length $E(Q)$. Now, imagine that we modulate the charge distribution on the string $Q(z)=Q_{0}+\delta \cos k z$, where $\delta \ll Q_{0}$. If we take $k$ to be large enough, then gradient energy from the $z$-modulation can be ignored, and at a given $z$ the string configuration resembles that of a uniformly charged string with charge per unit length $Q(z)$. Then, the change in energy due to the perturbation at $z$ can be approximated by

$\left.\delta E(z) \approx \frac{\mathrm{d} E}{\mathrm{~d} Q}\right|_{0} \delta \cos k z+\left.\frac{1}{2} \frac{\mathrm{d}^{2} E}{\mathrm{~d} Q^{2}}\right|_{0} \delta^{2} \cos ^{2} k z$,

where we have kept terms to second order in $\delta$. Averaged over one wavelength of the perturbation, this gives

$\left.\langle\delta E\rangle \approx \frac{\delta^{2}}{4} \frac{\mathrm{d}^{2} E}{\mathrm{~d} Q^{2}}\right|_{0}$

so that the energy of the string is lowered by the perturbation if

$\left.\frac{\mathrm{d}^{2} E}{\mathrm{~d} Q^{2}}\right|_{0}<0$

This condition is generally satisfied for the model we have studied above so that our strings are unstable to $z$ perturbations for large enough $k$.

An important feature of the instability is that it is an instability towards the growth of long wavelength modes in the charge distribution along the string. Hence it is irrelevant for loops or open segments that are smaller than the smallest wavelength of instability ${ }^{\# 2}$. The critical wavelength of instability decreases with increasing charge, but for $m=2$, and for the maximum value of charge that we have considered, the wavelength is always larger than several string widths. For still larger values of $m$, or for smaller values of the charge, the critical wavelength increases further and the clumping instability becomes less relevant. The addition of a repulsive force between the charges can also lead to reduced instability. An extreme case of this is when the force between charges is also mediated by gauge fields (spin 1). Here it can be shown that the resulting long range repulsive force can cause $\mathrm{d}^{2} E / \mathrm{d} Q^{2}$ to become positive for

\#2 In the cosmological electroweak phase transition, one expects the length distribution of loops and segments to be exponentially suppressed and so only small loops and segments are relevant. 
large enough charge so that the clumping instability is absent altogether $\# 3, \# 4$.

The most pertinent question at this juncture is if the standard electroweak model also admits stable bound electroweak strings. In this case one needs to look at fermionic and vector boson bound states with the experimentally determined parameters. The physical argument - in which we view the string as a bag - applies to fermions also and so fermionic bound states will also improve the stability of electroweak strings. One difference with the bosonic case is that fermions obey the Pauli exclusion principle and so every additional fermion that we put in the string bag must occupy a different quantum state. This makes it somewhat less energy efficient to pack fermions onto the string $\# 5$.

There are some additional (technical) difficulties in investigating the bound $Z$-string in a realistic setting. The first such difficulty is that the quarks and leptons carry electromagnetic charge and the electromagnetic field of the bound state must also be taken into account in the stability analysis. A second difficulty is that the stability analysis must necessarily include more than one fermion since the left-handed fermions occur in SU(2) doublets. Both these difficulties promise to make the realistic stability analysis an Herculean task.

The above analysis of the $Z$-string is a particular case of a very general prescription for finding possible stable solutions in any field theory. The first step in this prescription is to find embedded defect solutions ${ }^{\# 6}$ following the scheme in ref. [2] and the second step is to put charge on the defect and to see if it can be stabilized. In particular, one may ask if bound states can stabilize the electroweak $W$-string. It would also be of some interest to study the effects of bound states on embedded monopole configura-

\#3 Gauged non-topological solitons have been studied in ref. [16].

\#4 In fact, this is effectively what happens when we increase $m$. A larger $m$ means that the attractive Yukawa potential between $\chi$ charges is of shorter range and hence, the attraction between $\chi$ charges is reduced.

\#5 In both the bosonic and fermionic cases, the addition of more charge is expected to lead to diminishing returns in improved stability. This is because the string "swells" as we increase the charge and this costs energy.

\#6 The simplest example of an embedded defect is a domain wall in a global $U(1)$ model. tions [2]. Given the rather general stability analyses of monopoles $[17,18]$, it would be worthwhile to see how bound states can fail to stabilize the embedded monopole. Another unstable configuration that might be stabilized by the presence of bound states is the sphaleron [5].

During a phase transition in the early universe, we expect embedded string configurations to form. If the strings existed in a vacuum, they would be unstable towards spreading out radially and unwinding. However, we know that the strings at the phase transition are immersed in a thermal background and the dynamics is likely to be completely different from that in vacuum. If we consider a string of length $L$, the number of charged particles on the string will be proportional to $L$ while the net charge on the string will be proportional to $\sqrt{L}$. At temperatures close to the phase transition, particles will enter and leave the string but, on average, the number density of bound particles will go like $L / L=1$ and the charge density will go like $\sqrt{L} / L=1 / \sqrt{L}$. The stabilization of the string at such high temperatures will be related to the number density of particles - and not the charge density - on the string. (This is because there is a certain amount of energy that is saved for every particle that lives on the string, whether it be positively or negatively charged.) The number density of charges on the string around the time of the phase transition is quite high due to the large density of particles in the bath and the fact that the string acts like a potential well for the charges. Thus stabilization at these early times may be achievable.

What is the cosmological evolution of such strings? The evolution of the strings is necessarily tied to the evolution of the gas of particles bound to the string. As time goes on, the strings will try to shorten and straighten out. This exerts a pressure on the gas of particles bound to the string and would tend to raise the temperature of the gas. At the same time, the gas on the string can radiatively cool, leading to the annihilation of plus and minus charges and reducing the stability of the string. However, as the string length decreases the net charge density should become more concentrated on the string. We can envision two possible outcomes of this evolution: the charge density on the string could fall below the critical value required for stability, leading to the ultimate decay of the string; or else, a critical charge density could be 
maintained throughout the evolution and the string would either collapse or eventually reach a stable configuration (see below). The evolution will depend on the various parameters of the theory and it is not possible for us to be able to predict an outcome at this time. Note that this cosmological problem is quite similar to the problem of forming vortons [19] and non-topological solitons [20] in the early universe.

Finally we would like to make one more comment that is relevant for cosmology and the observational prospects for electroweak defects. A loop of electroweak string has two distinct instabilities: the first is the field-theoretic instability that we have discussed above and the second is a dynamical instability against collapse. In this letter we have argued that the presence of bound states on the string can protect the string against the field-theoretic instability. These bound states also add to the energy density of the string without correspondingly adding to the pressure. This implies that the dynamics of bound strings should be similar to that of wiggly strings [21] or to current carrying superconducting strings - depending on the nature of the charge on the string. It is also known from previous work that currents [11] on the string can protect the loop against dynamical collapse $[22,19,23,24]$. These facts together suggest the possibility that there are stable, static ring configurations (also, vortons [19]) in the standard electroweak model. If the sphaleron is also stabilized by bound states, it would imply additional particle-like solutions in the model.

We are grateful to Alex Vilenkin and Jose Wudka for discussions. T.V. acknowledges the support of the NSF and R.W. acknowledges the support of NASA Grant No. NAGW-2802.

\section{References}

[1] H.B. Nielsen and P. Olesen, Nucl. Phys. B 61 (1973) 45.

[2] T. Vachaspati and M. Barriola, Phys. Rev. Lett. 69 (1992) 1867

[3] T. Vachaspati, Phys. Rev. Lett. 68 (1992) 1977; Nucl. Phys. B, to be published.

[4] Y. Nambu, Nucl. Phys. B 130 (1977) 505.

[5] N.S. Manton, Phys. Rev. D 28 (1983) 2019.

[6] M. James, L. Perivolaropoulos and T. Vachaspati, Phys. Rev. D 46 (1992) R5232.

[7] W. Perkins, Phys. Rev. D 47 (1993) 5224.

[8] R. Holman, S. Hsu, T. Vachaspati and R. Watkins, Phys. Rev. D 46 (1992) 5352.

[9] T.D. Lee, Phys. Rep. C 23 (1976) 254.

[10] T. Vachaspati and A. Achúcarro, Phys. Rev. D 44 (1991) 3067.

[11] E. Witten, Nucl. Phy. B 249 (1985) 557.

[12] M. Hindmarsh, Semilocal Topological Defects, DAMTP preprint (1992).

[13] M. Hindmarsh, Phys. Rev. Lett. 68 (1992) 1263.

[14] A. Achúcarro, K. Kuijken, L. Perivolaropoulos and T. Vachaspati, Nucl. Phys. B 388 (1992) 45.

[15] E. Copeland, R. Kolb and K. Lee, Phys. Rev. D 38 (1988) 3023.

[16] K. Lee, J.A. Stein-Schabes, R. Watkins and L. Widrow, Phys. Rev. D 39 (1989) 1665.

[17] R. Brandt and F. Neri, Nucl. Phys. B 161 (1979) 253.

[18] S. Coleman, The Magnetic Monopole Fifty Years Later, Erice lectures 1981.

[19] R.L. Davis and E.P.S. Shellard, Nucl. Phys. B 323 (1989) 189;

R.L. Davis, Phys. Lett. B 207 (1988) 404; B 209 (1988) 485; Phys. Rev. D 38 (1980) 3722.

[20] J. Frieman, G. Gelmini, M. Gleiser and E. Kolb, Phys. Rev. Lett. 60 (1988) 2001.

[21] B. Carter, Phys. Rev. D 41 (1990) 3886; A. Vilenkin, Phys. Rev. D 41 (1990) 3038.

[22] E. Copeland, M. Hindmarsh and N. Turok, Phys. Rev. Lett. 58 (1987) 1910.

[23] C. Hill, H. Hodges and M. Turner, Phys. Rev. D 37 (1988) 263.

[24] A. Babul, T. Piran and D.N. Spergel, Phys. Lett. B 202 (1988) 307. 